\title{
Relationship between adverse childhood experiences and anxiety symptoms among Chinese adolescents: The role of self-compassion and social support
}

\author{
Xinli Chi ${ }^{1}$. Weiwei Jiang ${ }^{1} \cdot$ Tianyou Guo ${ }^{1}$ - Daniel L. Hall ${ }^{2,3} \cdot$ Christina M. Luberto $^{2,3} \cdot$ Liye Zou $^{1}$ \\ Accepted: 14 November 2021 \\ ๑) The Author(s), under exclusive licence to Springer Science+Business Media, LLC, part of Springer Nature 2021
}

\begin{abstract}
Previous studies have revealed that adverse childhood experiences (ACEs) create a significant and lasting effect of increased anxiety. However, few studies have examined the mediating and moderating mechanisms underlying this relation. The present study aimed to explore the mediating role of self-compassion and moderating role of social support in ACEs-anxiety relationship among Chinese adolescents. In this cross-sectional study, 1,764 middle school students completed questionnaires measuring their levels of ACEs, anxiety, self-compassion (SC), and social support. Correlations of variables were computed using Pearson's r. Mediation and moderated mediation models were tested using PROCESS macro with the regression bootstrapping method. After covariates (age and gender) were controlled, results showed that: (1) ACEs were positively associated with anxiety symptoms; (2) self-compassion partially mediated the ACEs-anxiety association; (3) both ACEs-anxiety and SC-anxiety relationships were moderated by social support. Specifically, social support was associated with lower anxiety, particularly among students with fewer ACEs or lower level of self-compassion. Reducing possible adversities existing in environment may help to reduce risk of anxiety for adolescents. Cultivating self-compassion is crucial for adolescents' mental health, since it may play a role in ACEs-anxiety relationship. Social support would operate as a buffer to ACEs in the relation with anxiety, under the circumstances of mild adversities, and a promoter to self-compassion in the relation with anxiety, under the circumstances of low self-compassion.
\end{abstract}

Keywords Adolescent $\cdot$ Adverse childhood experience $\cdot$ Anxiety symptom $\cdot$ Self-compassion $\cdot$ Social support

\section{Introduction}

Anxiety is the mind and body's reaction to stressful, dangerous, or unfamiliar situations. Anxiety symptoms consist of a series of emotional (e.g., unease, distress), cognitive (e.g., fears, worries, nervousness), physical (e.g., muscle tension, sweating), and behavioral (e.g., avoidance, overcompensation) changes (Vallance \& Fernandez, 2016). According to a

Liye Zou

liyezou123@szu.edu.cn

1 Exercise Psychophysiology Laboratory, Institute of KEEP Collaborative Innovation, Shenzhen University, Shenzhen 518060, China

2 Benson-Henry Institute for Mind Body Medicine, Massachusetts General Hospital, Boston, MA, USA

3 Department of Psychiatry, Harvard Medical School, Boston 02114, USA research review in 2015, anxiety is the most common mental health problem among adolescents, affecting approximately 117 million youth from 5 to 19 years around the world (Polanczyk et al., 2015). Results from another metaanalysis indicated the prevalence of anxiety disorders in children and adolescents globally has reached 3.1\%-17.5\% (Rockhill et al., 2010).

Additionally, the data from the National Institutes of Health of the United States has reported that the prevalence of anxiety disorder among American adolescents climbed 20\% between 2007 and 2012 (McCarthy, 2019). With regard to Chinese adolescent anxiety, several large-scale epidemiological studies reported prevalence ranges from $13.3 \%$ to 34.2\% (Liu \& Sun, 1997; Su, 2007; Yao et al., 2009; Zhang et al., 2013). Anxiety symptoms during adolescence may cause dysfunctions in academic performance, social ability, and/or family relations (Lin, 2009; Vallance \& Fernandez, 2016), and may trigger other psychological disorders such as depression (McCarthy, 2019) and suicidal behavior (Yang, 
2005). Moreover, the mental health problems may persist into adulthood (Keller et al., 1992; Ranøyen et al., 2018).

\section{Adverse Childhood Experiences and Adolescent Anxiety Symptoms}

According to the Bronfenbrenner's ecological system theory, the onset and the development of mental illness is greatly impacted by the negative relational systems including microsystem (e.g. poor family functioning), mesosystem (e.g. school maladjustment and poor peer relationship), exosystem (e.g. harsh parents' workplace), and macrosystem (e.g. depressed culture and social environment) (Bronfenbrenner, 1977; Noffsinger et al., 2012). Experiencing adverse childhood experiences (ACEs) in these relational systems is one of the most proximal risk factors to child and adolescent anxiety symptoms (Kemp et al., 2016; Rockhill et al., 2010). ACEs are potentially traumatic events that occur in childhood, such as experiencing violence, abuse, or neglect, witnessing violence in the home or community, and other aspects of children's environment that can undermine their sense of safety, stability, and bonding (National Center for Injury Prevention \& Control, 2020). The ACEs-anxiety association in adolescents has been well documented (Clark et al., 2010; Cuffe et al., 2005). For example, recent studies using data from 2016-2017 National Survey of Children's Health of the United States found that all ACE measures were significantly associated with higher odds of anxiety (Elmore \& Crouch, 2020), and children and adolescents with multiple ACEs were most likely to have anxiety symptoms (Lee et al., 2020). The above findings provided evidence to suggest that $\mathrm{ACE}$ is a risk factor underlying the development of anxiety in adolescents. Understanding how ACEs contribute to anxiety symptoms, and what protective factors might buffer this relationship, may be useful to inform prevention and treatment efforts for adolescent development.

\section{Self-Compassion as Mediator}

To better understand the associations between multiple risks factors and adolescent anxiety symptoms, the selfsystem model is a helpful conceptual framework ( $\mathrm{Li}$, 2012; Wolchik et al., 2008; Yang et al., 2013). The model proposes that multiple risk factors such as ACEs lead to the development of mental health problems through the pathways of decreasing or destructing the function of self-system beliefs or processes. Specifically, individuals actively construct themselves in the relation to their social context via self-system processes ( $\mathrm{Li}, 2012$; Sandler, 2001). If the surrounding environment or relationship is unfavorable, a negative self-system would be formed, such as low self-esteem, resilience, and coping skills. These psychological vulnerabilities will increase the risk of individual mental health problems. Previous studies of self-system model have included multiple positive psychological traits including gratitude, optimism, self-control, self-compassion, self-esteem and self-efficacy. (Judge et al., 2002; Li, 2012). As an important promotive factor of self-system model, self-compassion is indicated to be closely related to ACEs and psychological outcomes (van Dam et al., 2011). Selfcompassion entails three basic components: self-kindness - extending kindness and understanding to oneself, common humanity - seeing one's experiences as part of the larger human experience, and mindfulness - holding one's painful thoughts and feelings in balanced awareness (Neff, 2003b).

Various of types of ACEs such as low early warmth, parental rejection, and abuse have been associated with lower levels of self-compassion and higher levels of selfcriticism (Naismith et al., 2019; Pepping et al., 2015; Tanaka et al., 2011). Furthermore lower levels of self-compassion have been associated with greater anxiety symptoms (Neff, 2003a). For example, results from a meta-analysis $(n=12$ studies) reported that self-compassion was moderately associated with anxiety symptoms $(d=-0.51 ; p<0.001)$ (MacBeth \& Gumley, 2012). Likewise, previous studies indicated that self-compassion may serve as a mediator in the relationship between ACEs and negative psychological outcomes among adolescents. For example, a study in Spain including 109 adolescents found the participants who had experienced more types of victimization (e.g., exposure to violence) had lower levels of self-compassion, which further led to higher levels of psychological maladjustment including anxiety (Játiva \& Cerezo, 2014). To date, however, the mediating role of self-compassion on ACEs-anxiety among Chinese adolescents remains unexamined.

\section{Social Support as Moderator}

As one of the most frequently studied protective factors, social support usually refers to the functions performed for individual by significant others, such as family members, friends, and coworker (Thoits, 1995). The protective factor model under the framework of resilience theory suggested that a protective factor may modify the relationship between risk or promotive factors and psychological outcomes (Zimmerman, 2013). The model further described two possible protective models: risk-protective and protective-protective. The risk-protective model indicates that protective factors operate to reduce the negative effect of risk factors on psychological outcomes, and the protectiveprotective models operate to enhance the positive effect of promotive factors on outcomes. One approach is to test the two models by examining the interaction effects between risk/promotive and protective factors (Zimmerman, 2013). In other words, the protective factor (i.e., social support) may play a moderating role between the relation of risk 
factor (i.e., ACEs) and psychological outcome (i.e., anxiety symptoms) as well as the relation between promotive factor (i.e., self-compassion) and psychological outcome (i.e., anxiety symptoms).

Previous studies also provided evidence for the moderating role of social support. For example, a study based on 172 women who suffered childhood sexual abuse showed that increased levels of perceived social support were significantly predictive of lowered levels of PTSD symptoms (Hyman et al., 2003). Another longitudinal study on 135 clerical employees revealed that high levels of social support mitigated against the negative effects of high strain jobs on levels of job satisfaction and work performance (Sargent \& Terry, 2000). Regarding the interaction between self-compassion and social support, published studies were inadequate, especially in Chinese context. However, some literatures provided insight into the interaction of psychological assets and social support. For example, a study in Spain among 154 elderly people indicated that social support moderated the relation between self-efficacy and perceived mental health, such that a higher level of social support increased the positive effect of self-efficacy on perceived mental health (Lara et al., 2020).

However, under the framework of resilience theory, studies have found inconsistent results on the direction of regulation in interaction models. For example, conversely with the risk-protective model, some researchers found in the context of low level of adversities, adolescents with higher level of social support would have less risk of experiencing mental health problems, and in the context of higher level of adversities, adolescents with both higher and lower level of social support would display more mental health problems ( $\mathrm{Li}, 2012)$. At the high level of the risk, protective factors might lose their ability to resist the risk (Rutter, 1987; Vanderbilt-Adriance \& Shaw, 2008). Such results may be explained as vulnerability processes proposed by Rutter, which states that vulnerability and protectiveness are two opposite sides of the same coin, and individuals with high levels of a commonly believed protective factor could be more susceptible to risk factors under some circumstances (Rutter, 1987). Subsequently, a number of research findings supported the vulnerability process hypothesis (Gutman et al., 2003; Saegert \& Evans, 2003; Vanderbilt-Adriance \& Shaw, 2008; Winkel et al., 2009). Similarly, conflicting with the protective-protective model, in some previous studies the increasing levels of protective factors was found to weaken the association between promotive factors and outcomes instead of enhancing it (Reyes Fernández et al., 2014; Wang et al., 2009). This type of interactions has been named as antagonistic interactions by Cohen et al. (2003), which means that two positive factors with the same effect direction on outcomes could have an interaction effect with an opposite direction on outcomes. For example that ability and motivation are both positive factors to school achievement, but the effect of certain ability may be weakened by certain motivation (Cohen et al., 2003). This is explained that when individual resources (e.g., self-esteem and self-compassion) are high, social adaptation (e.g., low level of anxiety symptoms) may be almost always in a good state, and the beneficial effects of protective resource (e.g., social support) are not easily reflected (Li, 2012; Reyes Fernández et al., 2014).

\section{The Present Study}

The relationship between ACEs and anxiety symptoms has been extensively studied, but very few studies have explored the mediating role of self-compassion and moderating role of social-support in ACEs-anxiety relationship, especially among Chinese adolescents. Exploring these possible mediators and moderators is important to understand modifiable factors that influence the ACE-anxiety relationship and thus may serve as useful targets for future intervention research. The majority of previous ACEs studies have focused on adults. However a longitudinal study indicated that ACEs were more strongly associated with poor mental health of adolescence in relative to those adults (Clark et al., 2010), suggesting that more attention should be paid to the proximal effects of ACEs on adolescents (Hunt et al., 2017). China has 170 million adolescents, making it the worlds' second largest adolescent population (Luo et al., 2019), and the mental health problems of these Chinese adolescents are commonly reported. Data from the National Health Commission of China has indicated that approximately 30 million children and adolescents in China are suffering from various psychological disorders (Dai, 2018), including anxiety. This figure highlights the necessity and urgency of research on mental health and its associated risk and protection mechanisms in Chinese adolescents. In addition, in comparison with other stages of adolescence, junior middle school students may face more difficult challenges. They are in an unstable status during puberty in which they experience tremendous changes physically and psychologically. Furthermore, a big transition from primary to middle school has brought great academic and interpersonal pressure. ACEs significantly increase the possibility of internal conflicts and cause mental health problems like anxiety symptoms in junior middle school students (Hu \& Gan, 2017). Some Chinese researchers suggested that the psychological crises among junior middle school students are noticeable, which requires special attention-timely interventions are necessary to prevent mental disorders in future (Zhao et al., 2013). Therefore, a study exploring modifiable mediators and moderators of the ACE-anxiety relationship among Chinese adolescents, particularly among junior middle school students, would contribute not only to adolescent mental health, but also consolidate the theoretical framework of ACEs-anxiety relations and its protective mechanisms. 
Based on the aforementioned inconsistent model hypotheses and previous findings regarding moderation direction of a protective factor (e.g., social support), this study attempted to examine which theoretical models (risk-protective model $v s$. vulnerability process hypothesis; protective-protective model vs. antagonistic interaction hypothesis) would be supported by the present study findings. Specifically, the present study aimed to examine the relationship between ACEs, anxiety symptoms, self-compassion and social support among Chinese adolescents. From previous studies, the study hypothesized that self-compassion would play a mediation role between ACEs and anxiety symptoms (hypothesis 1), social support would moderate the relationship between ACEs and anxiety symptoms (hypothesis 2), as well as the relationship between self-compassion and anxiety symptoms (hypothesis 3 ). The proposed moderated mediation model is presented in Fig. 1.

\section{Methods}

\section{Participants and Power Calculation}

The data were collected in May 2020, in Yizhou district of Hechi city, Guangxi province. Guangxi is an economically underdeveloped province in southern China, and Hechi is a poverty-stricken area with about $20.15 \%$ population below the poverty line in 2015 (Wei, 2019). As mentioned in introduction that ACEs are closely related with poverty, there is more practical significance to investigate status of ACEs and adolescents' wellbeing in a poverty area like Hechi. The formula $n=\frac{\left(z^{2}\right) P(1-P)}{d^{2}}$ was applied to calculate an appropriate sample size to investigate the prevalence of ACEs in the district (Arya et al., 2012). According to previous study,

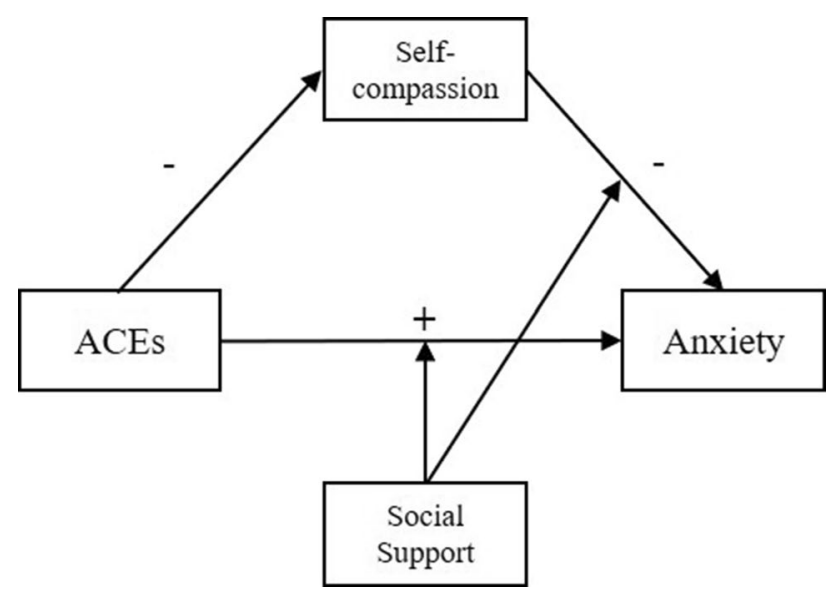

Fig. 1 Conceptual Model of Adverse Childhood Experiences Leading to Anxiety Symptoms via Self-Compassion, Moderated by Social Support. Note. ACEs $=$ adverse childhood experiences the prevalence of ACEs differs by regions and populations (Carlson et al., 2019), thus the expected prevalence $P$ was set to 0.5 , allowable error $d=0.03$. The expected sample size is calculated as 1067 . This study chose junior middle school students (grade 7 to 9) as participants. To reach the ideal sample size, 12 junior middle schools, each with 3 classes, were randomly selected to conduct the survey. To strike balance between the target sample (adolescents) and their comprehensibility/readability of questionnaires, students who reached age range of 12-17 were included. Meanwhile, the exclusion criteria were specified below: (a) students aged below 12 or 18 years and above; (b) with a history of mental illness, and (c) with a severe cognitive impairment and/or audio-visual impairment. Total 1833 students volunteered to participate in the study. After eliminating invalid questionnaires, a total of 1764 qualified responds remained. The participants comprised $801(45.41 \%)$ male students and 963 (54.59\%) female students, aged between 12 and 17 years old $(\mathrm{M}=14.24, \mathrm{SD}=0.96)$.

\section{Procedure}

With the assistance of local education authority, official approvals were obtained from the selected schools. Considering safety during the pandemic, questionnaires were distributed to students by school teachers through an online survey platform, in which instructions were described. The questionnaire contained scales of ACEs, anxiety symptoms, self-compassion, social support, and some sociodemographic information. Since the questionnaire was forced choice (i.e., required a response to each item before proceeding to the next page or submission), missing data were automatically excluded. Guardians of participants have been informed with investigation by head of teachers before data collection. The purpose of this study was explained to all participants and it was highlighted that all the collected data would be analyzed in an aggregated manner with personal information being kept in strict confidentiality. All participants were voluntary, all participants and guardians had signed an online consent form before filling the questionnaires. Recruitment and data collection procedures were approved by the Human Research Ethics Committee (No. 2020005) of Shenzhen University.

\section{Measurements}

Anxiety symptoms (dependent variable) were measured using the Chinese version of the Generalized Anxiety Disorder Scale (GAD-7), which was originally developed by Spitzer et al. (2006). The Chinese version has been studied with good reliability and validity among adolescents (Zhou et al., 2020). It is a 7-item scale that assessed the degree of individuals' generalized anxiety disorders (e.g., Being so 
restless that it's hard to sit still). All items are rated on a 4-point Likert-type scale $(0=$ "Not at all", $3=$ "Nearly every day"). The level of anxiety is reflected by a total score of the 7 items, higher scores indicate higher level of individuals' anxiety. The Cronbach's $\alpha$ coefficient of the scale in this study was 0.92 .

ACEs (independent variable) were measured using the revised Adverse Childhood Experiences Questionnaire (ACEQ-R) developed by Finkelhor and colleagues (2015). To ensure semantic accuracy in scale translation, this scale was first translated to Chinese, followed by back-translation. Three bilingual experts were invited to verify the translation along with 30 adolescents who engaged in a pre-test to determine its readability. The ACEQ-R assesses 14 aspects of adverse childhood experience: emotional abuse, physical abuse, sexual abuse, emotional neglect, physical neglect, domestic violence, family drug/alcohol problem, family mental illness, parental separation/divorce, parent ever go to prison, peer victimization, peer isolation, exposure to community violence, and low socioeconomic status. Response for each item was made by selecting either "yes" or "no". Responding "yes" will code as "1" and be considered to have experienced corresponding adversity; responded "no" will code as " 0 ". A total score of ACEQ-R summarized the 14 items scoring and thus ranged from 0 to 14 . The total scores represent number of different types of ACEs participants have experienced in childhood. The scale demonstrated good internal reliability $(\alpha=0.87)$ and good fit indices in confirmatory factor analysis $(\mathrm{CFI}=0.91, \mathrm{TLI}=0.90$, RMSEA $=0.08$, SRMR $=0.05)$ in this study.

Self-compassion (mediator) was measured using the Self-compassion Scale Short Form (SCS-SF) constructed by Neff and colleagues (Raes et al., 2011). To follow the standard procedures, this original version was first translated to Chinese, followed by back-translation. Second, three bilingual experts were invited to verify the translation along with 30 adolescents who engaged in a pre-test to determine its readability. The scale consists of 12 items within 6 domains: self-kindness (two questions), common humanity (two questions), mindfulness (two questions), self-judgment (two questions, reverse scoring), isolation (two questions, reverse scoring), and over-identification (two questions, reverse scoring). All items are rated on a 5-point Likerttype scale ( $1=$ "almost never", $5=$ "almost always"). Selfcompassion is expressed by mean value of total scores of all six sub-scales with higher scores indicating higher level of self-compassion. The scale demonstrated good internal reliability $(\alpha=0.90)$ and good fit indices in confirmatory factor analysis $(\mathrm{CFI}=0.95, \mathrm{TLI}=0.91$, $\mathrm{RMSEA}=0.09$, $\mathrm{SRMR}=0.05$ ) in this study.

The Chinese version Social Support Rating Scale (SSRS) was used in this study to assess level of social support (moderator) among Chinese adolescents (Xiao, 1994). The SSRS consists of 10 items divided into three dimensions: objective support (three items), subjective support (four items) and support utilization (three items). All items are rated on a 4-point Likert scale ( $1=$ "none", 2 = "slight", 3 = "moderate", 4 = "great"). The total scores summarizing all items are used to assess the current social support status of individuals, higher scores indicate higher level of individuals' social support. The scale has been widely tested in Chinese context, and its reliability and validity have been shown good in previous studies among Chinese adolescents ( $\mathrm{Li}, 2004$; Liu et al., 2007). The Cronbach's $\alpha$ coefficient of the scale in this study was 0.88 .

\section{Statistical Analyses}

Analyses were conducted via SPSS (version 21). Mean and standard deviation (SD) were calculated for ACEs, self-compassion, social support and anxiety symptoms. Correlations were computed using Pearson's r. Mediation and moderated mediation models were using PROCESS (Hayes, 2013) with 5000-iteration bootstrapping to compute standardized Betas with $95 \%$ bias-corrected confidence intervals. Confidence intervals not containing zero are statistically significant.

\section{Results}

\section{Descriptive Statistics and Correlations Analysis}

The ACEs score was treated as a continuous variable in this study. The prevalence of individual categories of ACEs among participated adolescents were displayed in Table 1. The most frequently happened ACEs in this study are peer isolation/rejection (23.41\%), parents' emotional abuse (20.92\%), and family emotional neglect (17.23\%). Family mental illness $(4.14 \%)$, family drug/alcohol problem (4.14\%), and physical neglect (4.48\%) showed lowest prevalence rate. The ACEs score ranged from 0 to $14,54.54 \%$ of the participants reported 0 and $7.20 \%$ of the participants reported more than 5 .

Table 2 shows the results of descriptive statistics (mean and SD) and correlation analysis for major variables. As expected, the correlations between each two variables of anxiety, ACEs, self-compassion, and social support were significant. ACEs were positively associated with anxiety, and negatively associated with self-compassion and social support. Self-compassion was negatively correlated with anxiety and positively correlated with social support. Social support was negatively correlated with anxiety. Gender was significantly associated with anxiety and self-compassion. Age had a slight positive association with anxiety. 
Table 1 Prevalence of Individual Categories of ACEs

\begin{tabular}{llr}
\hline Individual Categories of ACEs & $\mathrm{n}$ & \multicolumn{1}{l}{$\%$} \\
\hline 1. Emotional abuse & 369 & $20.92 \%$ \\
2. Physical abuse & 132 & $7.48 \%$ \\
3. Sexual assault & 86 & $4.88 \%$ \\
4. Emotional neglect & 304 & $17.23 \%$ \\
5. Physical neglect & 79 & $4.48 \%$ \\
6. Parents' divorce/separation & 193 & $10.94 \%$ \\
7. Mother treated violently & 98 & $5.56 \%$ \\
8. Family drug/alcohol problem & 73 & $4.14 \%$ \\
9. Family mental illness & 73 & $4.14 \%$ \\
10. Parent ever go to prison & 114 & $6.46 \%$ \\
11. High peer victimization & 110 & $6.24 \%$ \\
12. High peer social isolation & 413 & $23.41 \%$ \\
13. High exposure to community violence & 90 & $5.10 \%$ \\
14. Low socioeconomic status & 183 & $10.37 \%$ \\
At least one category of ACEs & 802 & $45.46 \%$ \\
\hline
\end{tabular}

$\mathrm{N}=1764$

ACEs adverse childhood experiences

\section{Mediation model}

As demonstrated in Table 3 and Fig. 2, after controlling gender and age, ACEs were positively associated with anxiety in the absence of the mediator $(\beta=0.27, p<0.001)$. In addition, ACEs were negatively correlated with self-compassion $(\beta=-0.21, p<0.001)$. When ACEs were controlled for, self-compassion was negatively correlated with anxiety ( $\beta=-0.38, p<0.001)$. Next, self-compassion was included, and the positive association between ACEs and anxiety was still significant $(\beta=0.19, p<0.001)$. Finally, as shown in Table 4 , the indirect effect of self-compassion was $\beta=0.08$, and its $95 \%$ confidence interval was $[0.06,0.10]$, suggesting that self-compassion partially mediated the association between ACEs and anxiety, thus the hypothesis 1 was supported. Using indirect effect divided by total effect to calculate the effect ratio, the mediation effect accounted for $29.63 \%$ of the total effect of the association between ACEs and anxiety.

Table 2 Descriptive Statistics and Correlation Analyses

\begin{tabular}{lclllllll}
\hline 1 & $\mathrm{M} \pm \mathrm{SD}$ & Minimum Value & Maximum Value & 1 & 2 & 3 & 4 & 5 \\
\hline 1. Gender & - & - & - & 1 & & & & \\
2. Age & $14.24 \pm 0.96$ & 12 & 17 & $-0.05^{*}$ & 1 & & & \\
3. Anxiety & $3.91 \pm 4.65$ & 0.00 & 21.00 & $0.14^{* * *}$ & $0.08^{* * *}$ & 1 & & \\
4. ACEs & $1.31 \pm 2.41$ & 0.00 & 14.00 & -0.03 & -0.06 & $0.26^{* * *}$ & 1 & \\
5. Self-compassion & $3.15 \pm 0.45$ & 1.25 & 5.00 & $-0.10^{* * *}$ & -0.02 & $-0.43^{* * *}$ & $-0.21^{* * *}$ & 1 \\
6. Social support & $40.03 \pm 8.47$ & 12.00 & 66.00 & 0.03 & -0.01 & $-0.27^{* * *}$ & $-0.26^{* * *}$ & $0.36^{* * *}$ \\
\hline
\end{tabular}

All data is standardized, the beta values are standardized regression coefficients

Gender code $0=$ male; $1=$ female; ACEs $=$ adverse childhood experiences

${ }^{*} p<0.05, * * * p<0.001$

Table 3 Mediation model analysis

\begin{tabular}{|c|c|c|c|c|c|c|}
\hline \multirow[t]{2}{*}{ Predictors } & \multicolumn{2}{|l|}{$\begin{array}{l}\text { Model } 1 \\
\text { (Anxiety) }\end{array}$} & \multicolumn{2}{|c|}{$\begin{array}{l}\text { Model } 2 \\
\text { (Self-compassion) }\end{array}$} & \multicolumn{2}{|l|}{$\begin{array}{l}\text { Model } 3 \\
\text { (Anxiety) }\end{array}$} \\
\hline & $\beta$ & $\mathrm{t}$ & $\beta$ & $\mathrm{t}$ & $\beta$ & $\mathrm{t}$ \\
\hline Constant & $-0.17^{* * *}$ & -4.92 & $0.11^{* * *}$ & 3.21 & $-0.12^{* * * *}$ & -3.96 \\
\hline Gender & $0.31^{* * *}$ & 6.82 & $-0.21^{* * *}$ & -4.55 & $0.23^{* * *}$ & 5.45 \\
\hline Age & $0.09^{* * * *}$ & 4.08 & -0.03 & -1.12 & $0.84^{* * *}$ & 3.95 \\
\hline ACEs & $0.27^{* * *}$ & 11.91 & $-0.21^{* * * *}$ & -9.04 & $0.19^{* * *}$ & 8.90 \\
\hline Self-compassion & & & & & $-0.38^{* * * *}$ & -17.78 \\
\hline $\mathrm{R}^{2}$ & 0.10 & & 0.05 & & 0.24 & \\
\hline $\mathrm{F}$ & $65.60^{* * *}$ & & $33.48^{* * *}$ & & $137.06^{* * *}$ & \\
\hline
\end{tabular}

All data is standardized, the beta values are standardized regression coefficients ACEs adverse childhood experiences *** $p<0.001$ 


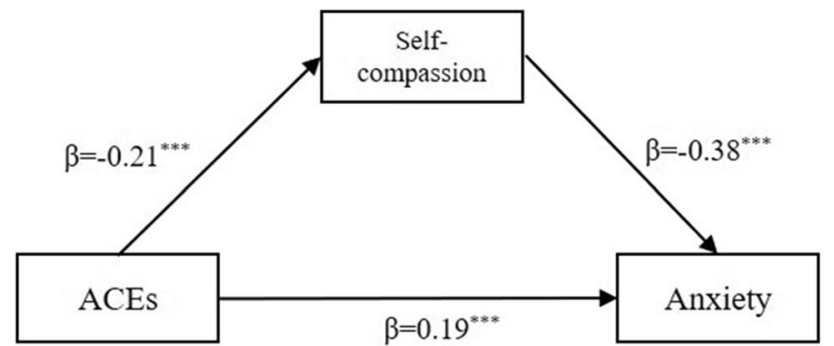

Fig. 2 direct and indirect effects of ACEs on anxiety. Note. The beta values are standardized regression coefficients; ${ }^{* * *} p<0.001$

\section{Testing for Moderated Mediation Model}

As described in Fig. 1, we hypothesized that social support would moderate the direct and indirect association between ACEs and anxiety.
Table 5 displayed the results of the conditional process analysis. As can be seen from the dependent variable model that predicts anxiety, after controlling gender and age, ACEs were positively associated with anxiety $(\beta=0.19, p<0.001)$, and the interaction between ACEs and social support was positively associated with anxiety $(\beta=0.06, p<0.05)$. Namely, social support moderated the relationship between ACEs and anxiety, such that the hypothesis 2 was supported. In addition, self-compassion was negatively associated with anxiety $(\beta=-0.35, p<0.001)$, although the interaction between self-compassion and social support was positively associated with anxiety $(\beta=0.09, p<0.001)$. It indicated that social support moderated the relationship between selfcompassion and anxiety, the hypothesis 3 was supported. The results revealed that the relationships between both ACEs and anxiety as well as self-compassion and anxiety were moderated by social support.

To explicate the moderating effect of social support, the plot of relationship between ACEs and anxiety at two levels
Table 4 Bootstrap results for mediation effect

\begin{tabular}{lllllc}
\hline & $\beta$ & Boot SE & LL 95\%CI & UL 95\%CI & Relative effect ratio \\
\hline Total Effect & 0.27 & 0.02 & 0.23 & 0.31 & $100.00 \%$ \\
Direct Effect & 0.19 & 0.02 & 0.15 & 0.23 & $70.37 \%$ \\
Indirect Effect & 0.08 & 0.01 & 0.06 & 0.10 & $29.63 \%$ \\
\hline
\end{tabular}

All data is standardized, the beta values are standardized regression coefficients. bootstrap sample size $=5000$.

ACEs adverse childhood experiences; $L L$ low limit, $U L$ Upper limit, $C I$ confidence interval

\begin{tabular}{|c|c|c|c|c|}
\hline Dependent variable model for predicting anxiety & $\beta$ & SE & $\mathrm{t}$ & $p$ \\
\hline Constant & $-0.13^{* * *}$ & 0.03 & -4.20 & $<0.001$ \\
\hline Gender & $0.23^{* * *}$ & 0.04 & 5.36 & $<0.001$ \\
\hline Age & $0.08^{* * *}$ & 0.02 & 3.96 & $<0.001$ \\
\hline ACEs & $0.19^{* * *}$ & 0.02 & 7.92 & $<0.001$ \\
\hline Self-compassion & $-0.35^{* * *}$ & 0.02 & -15.56 & $<0.001$ \\
\hline Social Support & $-0.10^{* * * *}$ & 0.02 & -4.42 & $<0.001$ \\
\hline ACEs $\times$ Social Support & $0.06^{*}$ & 0.02 & 2.50 & $<0.05$ \\
\hline Self-compassion $\times$ Social Support & $0.09^{* * *}$ & 0.02 & 4.45 & $<0.001$ \\
\hline Conditional direct effect at values of the moderator & $\beta$ & SE & LLCI & ULCI \\
\hline $\mathrm{M}-1 \mathrm{SD}$ & $0.13^{* * *}$ & 0.02 & 0.09 & 0.18 \\
\hline Mean & $0.19^{* * *}$ & 0.02 & 0.15 & 0.24 \\
\hline $\mathrm{M}+1 \mathrm{SD}$ & $0.25^{* * *}$ & 0.04 & 0.17 & 0.33 \\
\hline Conditional indirect effect at values of the moderator & $\beta$ & Boot SE & Boot LLCI & Boot ULCI \\
\hline $\mathrm{M}-1 \mathrm{SD}$ & 0.09 & 0.01 & 0.07 & 0.12 \\
\hline Mean & 0.07 & 0.01 & 0.06 & 0.09 \\
\hline $\mathrm{M}+1 \mathrm{SD}$ & 0.06 & 0.01 & 0.04 & 0.07 \\
\hline
\end{tabular}

All data is standardized, the beta values are standardized regression coefficients. bootstrap sample size $=5000$

ACEs adverse childhood experiences, $L L$ low limit, $U L$ upper limit, $C I$ confidence interval ${ }^{*} p<0.05,{ }^{* * *} p<0.001$ 


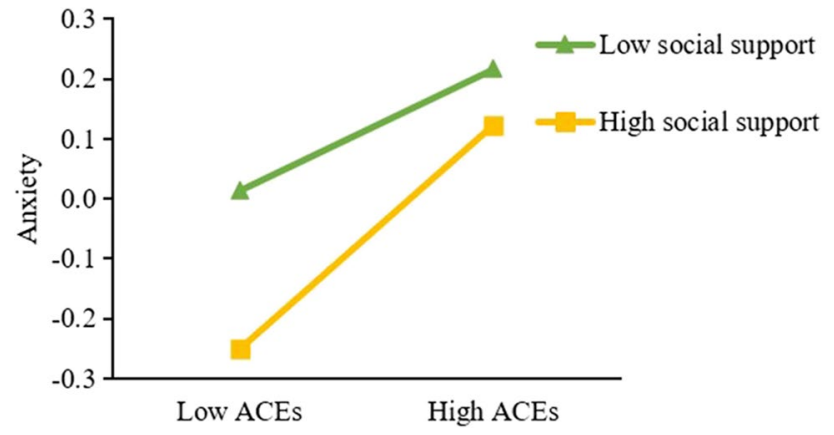

Fig. 3 Plot of the relationship between ACEs and anxiety at different levels of social support. Note. ACEs $=$ adverse childhood experiences

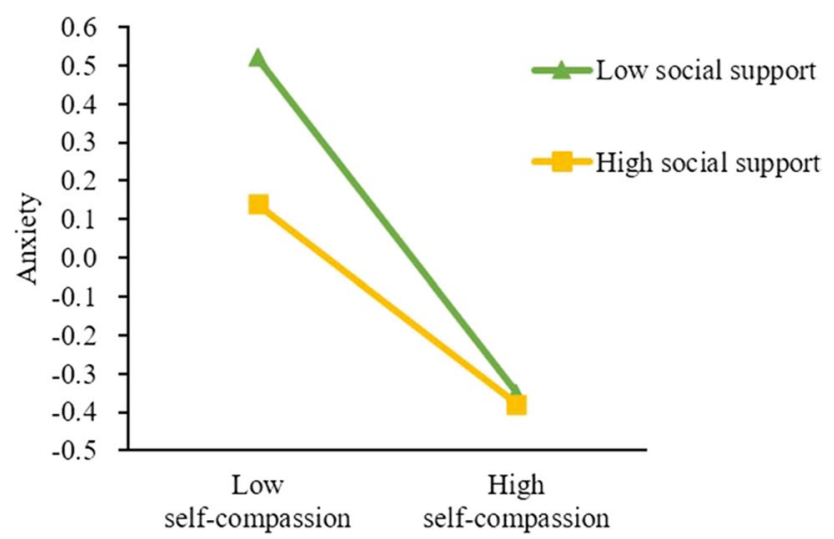

Fig. 4 Plot of the relationship between self-compassion and anxiety at different levels of social support

of social support (1 SD below the mean and $1 \mathrm{SD}$ above the mean) was described in Fig. 3. As can be seen from Fig. 3 and the conditional direct effect analysis in Table 5, for adolescents with high social support (1 SD above the mean), there was a strong positive association $(\beta=0.25$, $p<0.001$ ) between ACEs and anxiety. For adolescents with low social support (1 SD blow the mean), the association between ACEs and anxiety was still significant and positive but weaker $(\beta=0.13, p<0.001)$.

In addition, the plot of relationship between self-compassion and anxiety at two levels of social support (1 SD below the mean and $1 \mathrm{SD}$ above the mean) was displayed in Fig. 4. As can be seen from Fig. 4, for adolescents with low social support (1 SD blow the mean), the negative association between self-compassion and anxiety was stronger than adolescents with high social support (1 SD above the mean). Furthermore, as can be seen from the conditional indirect effect analysis in Table 5, the indirect effect of ACEs on anxiety through self-compassion was observed when social support was at a low level $(\beta=0.09)$, as well as when social support was at a high level $(\beta=0.06)$.

\section{Discussion and Conclusion}

\section{Discussion}

In the present study, we proposed a moderated mediation model to examine the relationship between ACEs, anxiety symptoms, self-compassion, and social support. Our findings have both theoretical and clinical implications for the prevention and treatment of anxiety symptoms among adolescents who have been exposed to trauma early in their lives, as detailed below.

Approximately half $(45.46 \%)$ of adolescents reported at least one type of ACEs in this study, which is at a lower level in relative to the ACEs prevalence (50-70\%) reported in previous studies of Western countries (Bynum et al., 2010; Jones et al., 2019; Merrick et al., 2018; Oral et al., 2016). Similarly, some previous studies in the United States also found that Asian Americans reported significantly lower ACEs prevalence than other ethnic groups (Bethell et al., 2017; Ye \& Reyes-Salvail, 2014). A possible explanation is that adolescents' judgments about whether they have been maltreated may be influenced by their culture. Studies showed that Asian societies are more tolerant to strict parental discipline and punishment than western societies (Lansford et al., 2005; Wang \& Liu, 2014). Under this circumstance, some adolescents who have been punished or reprimanded in their families may not consider their parents' behavior as a sort of "abuse". Moreover, the collective Chinese culture encourages interpersonal harmony, some researchers suggested that adolescents living this culture may reduce reporting conflicts and adversity experiences to avoid social stigma (Ye \& Reyes-Salvail, 2014). Although the prevalence in our study is lower than those in Western countries, the figure is still quite high (45.46\%). The finding implies that ACEs is very common among Chinese youth groups and that particular attentions should be paid to groups with ACEs. Additionally, in the present study, a significant relationship was observed between ACEs and adolescent anxiety symptoms. The finding supported the ecological system theory showing ACEs are detrimental factors of adolescent development in a relational system (Bronfenbrenner, 1977; Noffsinger et al., 2012) and is consistent with previous studies (Kemp et al., 2016; Rockhill et al., 2010). The results may be explained from cognitive and biological point of view. Cognitively, uncontrollable negative life events may increase adolescent perceptions of uncontrollability and expectations of negative events in future life, thus escalating their psychological vulnerability for anxiety states (Chorpita \& Barlow, 1998). Biologically, early life trauma is considered a possible cause for persistent sensitization of central nervous system, which may lead to 
the hyperactivity of corticotropin-releasing factor (CRF) systems as well as alterations in other neurotransmitter systems, resulting in increased vulnerability to the development of mental disorders such as anxiety symptoms (Binder \& Nemeroff, 2010; Heim \& Nemeroff, 2001).

Our findings also shed light on the role of self-compassion, which accounted for $29.63 \%$ of the effect of ACEs on adolescents' anxiety symptoms. This result supported the self-system model, which proposes ACEs may lead to mental health problems (e.g., anxiety symptoms) through the pathways of decreasing the function of adaptive self-system processes. From a cognitive perspective, a harmful environment or ACEs would contribute to the development of a stable negative cognitive style, which may increase self-criticism, negative self-attitudes and a lack of self-compassion (Neff \& McGehee, 2010). From the biological perspective, self-compassion is linked with the soothing system (Gilbert \& Procter, 2006; Naismith et al., 2019; Neff, 2012). The early years of life is essential for activation and maturation of the soothing system, whereas adversities like parental neglect and abuse may hinder its development and even cause problems in brain maturation (Gerhardt, 2006). This may further influence self-compassion development and overall mental health. From the developmental perspective, adolescence is the period to establish one's self-concept, such that ACEs during this period could be incorporated into adolescents' self-appraisals and lead to self-criticism and a negatively viewed sense of self (Rankin et al., 2004). Additionally, adolescents tend to be egocentric and hold personal fables, therefore they may over-dramatize their problems and interpret their negative experiences to be unusual (Neff \& McGehee, 2010). All these adolescent traits may lead to lower levels of self-compassion and poorer mental health in the context of ACEs. Taken together, findings in this study contributed to better understanding of possible pathways between ACEs and anxiety symptoms in Chinese adolescents, and highlighted the importance of self-compassion. Neff considered adolescence to be the period of life with lowest self-compassion (Kristin Neff, 2003a, b), and adolescents with high ACEs could be particularly vulnerable, especially for the junior middle school students who are facing dramatic physical and psychological changes of puberty. Meanwhile, benefited by the high psychological plasticity of this period, adolescence can also be the "window of opportunity" for cultivating self-compassion and forming a positive personality (Bluth et al., 2018). Thus, protection for adolescents from ACEs and intervention for adolescents with ACEs to promote self-compassion seem to be equally important for their mental health.

Finally, our findings substantiated the moderation role of social support in the relationship between ACEs, self-compassion and anxiety. This result enriched our understanding of the protective factor model based on resilience theory. The study found although social support was negatively associated with ACEs and anxiety, the interaction of ACEs and social supports was positively associated with anxiety. The positive association between ACEs and anxiety symptoms was stronger for adolescents with high social support. It can be explained that adolescents with low social support always have a high level of anxiety regardless the level of ACEs, thus the adolescents with high social support seemed to be more sensitive to the influence of ACEs. In other words, the protective effect of social support diminished as ACEs increases. This finding supported the vulnerability process hypothesis (Rutter, 1987), but not the risk-protective model (Zimmerman, 2013). Similar effects have been reported in previous studies (Gutman et al., 2003; Saegert \& Evans, 2003; Vanderbilt-Adriance \& Shaw, 2008; Winkel et al., 2009). These indicated that lack of social support created vulnerability. Enhancing social support is always beneficial and necessary. However, social support showed limitations on the degree to which it benefits mental health when facing major stressful events such as high level of ACEs (Thoits, 1995). In this situation, professional intervention or treatment become necessary for the recovery.

Although self-compassion and social support were both negatively associated with anxiety, the interaction of self-compassion and social support was positively associated with anxiety. The adolescents with low social support seemed to be more sensitive to the influence of self-compassion, and the sensibility receded while social support increased. Interestingly, high self-compassion adolescents presented a stable state of low anxiety, regardless low or high social support. It indicated self-compassion seemed to be a more powerful promotive factor to anxiety symptoms. This finding was consistent with the antagonistic interaction hypothesis (Cohen et al., 2003), but not the protective-protective model (Zimmerman, 2013, p. 382). It was explained that when promotive resources could be in a state of saturation (e.g., high level of self-compassion), anxiety symptoms always showed a "floor effect" under this circumstance. As a result, positive effects of protective resource (e.g., social support) are not easily played out (Li, 2012; Reyes Fernández et al., 2014). It indicated that for adolescents with low selfcompassion, enriching social support would be an effective way to improve their psychological well-being; and cultivating self-compassion would always be beneficial for adolescents, regardless of low or high social support.

Notably, the moderation effects of social support in ACEs-anxiety and SC-anxiety relationships were inconsistent with the classic protective factor model. Although it can be explained by the vulnerability process and antagonistic interaction, adolescents' developmental characteristics may be another factor in response to results of this study. According to Elkind (1967), adolescents tend to exhibit egocentrism, which means they may distort their perceptions of 
self and others in a self-centered way. Particularly for junior middle school students who are facing the pressure of academic transition and puberty changes, they may behave in ways that are distancing, opinionated, and overly defensive (Steinberg, 2011). Additionally, many Chinese researchers discussed the closed-mindedness of adolescents (Lin, 2009; Wang, 2006), which could be a form of egocentrism influenced by the more introvertive Chinese culture. These characteristics may prevent adolescents from perceiving social support, thus diminish its protective effect, especially under the high ACEs condition. However, owing to the cross-sectional study design in a single country, future studies (e.g., longitudinal studies and cross-cultural studies) may confirm or negate these findings.

\section{Limitations and Directions for Future Research}

Firstly, this study utilized a cross-sectional design which allowed us to determine correlations but not a causal association. Further longitudinal studies are needed to better understand the relationship between variables. Secondly, the data was collected during the first three weeks that middle school students return to school after the COVID-19 lockdown in China, which is a special period of time that students facing pressure from both academic and social uncertainty. There is reason to assume that the general level of adolescents' anxiety was raised up in this study. Further studies should take more control variables like life events into consideration. Thirdly, all the participants in this study are from one district of Hechi city, Guangxi province of China, it limited the sample representativeness. To extend the survey to a national sample is necessary in further studies. Fourthly, all data in this study were collected through self-reported scales, bias may exist due to social desirability effect and/or memory errors. Particularly for the levels of anxiety symptoms, the scores from GAD-7 are not equivalent to clinical diagnoses. Objective measures and diagnostic interviews could be considered in further studies. Finally, the questionnaires used in this study were forced choice, which means that participants were not allowed to skip any questions. That may cause bias in results if participants were uncomfortable with some of the questions. The form of questionnaire could be improved in further studies.

\section{Conclusion}

The study tested the mediating role of self-compassion and the moderating role of social support in the relationship between ACEs and anxiety. The results contribute to a more detailed understanding of how ACEs may influence anxiety in adolescents, providing evidence-based mechanistic targets for future longitudinal research. This study has significant theoretical and practical implications. Theoretically, the results enriched our understanding of the protective factor model based on the resilience theory. Specifically, a protective factor could magnify the negative correlation of risk factors and psychological outcomes while reducing the positive correlation of promotive factors and outcomes, under certain conditions like this study. It suggests that more different effect patterns could exist in the interactions of risk factors and protective factors under the resilience framework. Practically, this study provided specific implications for Chinese adolescent protection and mental health efforts. First, it suggests that to reduce risk of anxiety and enhance psychological well-being for adolescents, parents and educators should work to reduce possible adversities that exist in families (e.g., lack of warmth) and school settings (e.g., peer isolation). Programmatic efforts in school such as antibullying initiatives would be beneficial. Secondly, cultivating self-compassion is also crucial for adolescents' mental health, since it may play a role in how ACEs lead to anxiety problems. Incorporating self-compassion programs into school mental health education, or introducing self-compassion therapies to adolescents in need, may be advantageous for adolescents' mental health development. Furthermore, a program focusing on compassion broadly could be of benefit, since self-compassion is more difficult than compassion for others for many people. Starting with compassion for others could help minimize bullying and provide a foundation for learning to apply compassion to the self - targeting both important goals of reducing bullying and increasing self-compassion. At last, social support would operate as a buffer to ACEs in the relation with anxiety, under the circumstances of mild adversities, while social support would operate a promoter to self-compassion in the relation with anxiety, under the circumstances of low selfcompassion. Overall, a high level of social support is always beneficial for adolescents' mental health. However, to provide support in a more effective way, educators and relevant specialists should take possible risk and promotive factors like ACEs and self-compassion into consideration.

Acknowledgements The authors are extremely grateful to all the participants who took part in this study.

Funding This paper was supported by fund for research of cultivating innovative practical ability of youth in Shenzhen (grant number ybzz19009).

Data Availability The datasets generated during and/or analyzed during the current study are available from the corresponding author on reasonable request.

\section{Declarations}

Conflict of Interest The authors declare that the research was conducted in the absence of any commercial or financial relationships that could be construed as a potential conflict of interest. 


\section{References}

Arya, R., Antonisamy, B., \& Kumar, S. (2012). Sample size estimation in prevalence studies. Indian Journal of Pediatrics, 79(11), 1482-1488. https://doi.org/10.1007/s12098-012-0763-3

Bethell, C. D., Davis, M. B., Gombojav, N., Stumbo, S., \& Powers, K. (2017). A national and across-state profile on adverse childhood experiences among U.S. Children and possibilities to heal and thrive. Johns Hopkins Bloomberg School of Public Health. http:// www.cahmi.org/projects/adverse-childhood-experiences-aces/.

Binder, E. B., \& Nemeroff, C. B. (2010). The crf system, stress, depression and anxiety-insights from human genetic studies. Molecular Psychiatry, 15(6), 574-588. https://doi.org/10.1038/mp.2009.141

Bluth, K., Mullarkey, M., \& Lathren, C. (2018). Self-compassion: A potential path to adolescent resilience and positive exploration. Journal of Child and Family Studies, 27(9), 3037-3047. https:// doi.org/10.1007/s10826-018-1125-1

Bronfenbrenner, U. (1977). Toward an experimental ecology of human development. American Psychologist.

Bynum, L., Griffin, T., Riding, D. L., Wynkoop, K. S., Anda, R. F., Edwards, V. J., Strine, T. W., Liu, Y., McKnight-Eily, L. R., \& Croft, J. B. (2010). Adverse childhood experiences reported by adults - five states, 2009. MMWR. Morbidity and Mortality Weekly Report, 59(49), 1609-1613.

Carlson, J. S., Yohannan, J., Darr, C. L., Turley, M. R., Larez, N. A., \& Perfect, M. M. (2019). Prevalence of adverse childhood experiences in school-aged youth: A systematic review (1990-2015). International Journal of School \& Educational Psychology, 1-22.https://doi.org/10.1080/21683603.2018.1548397

Chorpita, B. F., \& Barlow, D. H. (1998). The development of anxiety: The role of control in the early environment. Psychological Bulletin, 124(1), 3-21. https://doi.org/10.1037//0033-2909.124.1.3

Clark, C., Caldwell, T., Power, C., \& Stansfeld, S. A. (2010). Does the influence of childhood adversity on psychopathology persist across the lifecourse? A 45-year prospective epidemiologic study. Annals of Epidemiology, 20(5), 385-394. https://doi.org/ 10.1016/j.annepidem.2010.02.008

Cohen, J., Cohen, P., West, S. G., \& Aiken, L. S. (2003). Applied multiple regression/correlation analysis for the behavioral sciences (Third edition). Lawrence Erlbaum Associates, Publishers. https:// doi.org/10.4324/9781410606266

Cuffe, S. P., McKeown, R. E., Addy, C. L., \& Garrison, C. Z. (2005). Family and psychosocial risk factors in a longitudinal epidemiological study of adolescents. Journal of the American Academy of Child and Adolescent Psychiatry, 44(2), 121-129. https://doi. org/10.1097/00004583-200502000-00004

Dai, L. (2018, May 25). 30 million adolescents under the age of 17 in china have emotional disorders, timely interventions are required. Beijing Evening News. https://www.takefoto.cn/viewnews-14795 14.html

Elmore, A. L., \& Crouch, E. (2020). The association of adverse childhood experiences with anxiety and depression for children and youth, 8 to 17 years of age. Academic Pediatrics, 20(5), 600-608. https://doi.org/10.1016/j.acap.2020.02.012

Elkind D. (1967). Egocentrism in adolescence. Child development, 38(4), 1025-1034

Finkelhor, D., Shattuck, A., Turner, H., \& Hamby, S. (2015). A revised inventory of Adverse Childhood Experiences. Child abuse \& neglect, 48, 13-21. https://doi.org/10.1016/j.chiabu.2015.07.011

Gerhardt, S. (2006). Why love matters: How affection shapes a baby's brain (Vol. 9). https://doi.org/10.1080/13698030601074476

Gilbert, P., \& Procter, S. (2006). Compassionate mind training for people with high shame and self-criticism: Overview and pilot study of a group therapy approach. Clinical Psychology \& Psychotherapy, 13(6), 353-379. https://doi.org/10.1002/cpp.507
Gutman, L. M., Sameroff, A. J., \& Cole, R. (2003). Academic growth curve trajectories from 1st grade to 12th grade: Effects of multiple social risk factors and preschool child factors. Developmental Psychology, 39(4), 777-790. https://doi.org/10.1037/ 0012-1649.39.4.777

Hayes, A. F. (2013). Introduction to mediation, moderation, and conditionalprocess analysis: A regression-based approach. The Guilford Press.

Heim, C., \& Nemeroff, C. B. (2001). The role of childhood trauma in the neurobiology of mood and anxiety disorders: Preclinical and clinical studies. Society of Biological Psychiatry, 49, 1023-1039. https://doi.org/10.1016/S0006-3223(01)01157-X(BiologicalPsych iatry,49(12),1023-1039)

Hu, W., \& Gan, X. (2017). Structure model of influencing factors between psychological abuse and mental health in the middle school students. Modern Preventive Medicine, 44(9), 1664-1667.

Hunt, T. K. A., Slack, K. S., \& Berger, L. M. (2017). Adverse childhood experiences and behavioral problems in middle childhood. Child Abuse \& Neglect, 67, 391-402. https://doi.org/10.1016/j. chiabu.2016.11.005

Hyman, S. M., Gold, S. N., \& Cott, M. A. (2003). Forms of social support that moderate ptsd in childhood sexual abuse survivors. Journal of Family Violence, 18(5), 295-300. https://doi.org/10. 1023/A: 1025117311660

Játiva, R., \& Cerezo, M. A. (2014). The mediating role of self-compassion in the relationship between victimization and psychological maladjustment in a sample of adolescents. Child Abuse \& Neglect, 38(7), 1180-1190. https://doi.org/10.1016/j.chiabu.2014.04.005

Jones, C. M., Merrick, M. T., \& Houry, D. E. (2019). Identifying and preventing adverse childhood experiences: Implications for clinical practice. JAMA. Advance online publication. https://doi.org/ 10.1001/jama.2019.18499

Judge, T. A., Erez, A., Bono, J. E., \& Thoresen, C. J. (2002). Are measures of self-esteem, neuroticism, locus of control, and generalized self-efficacy indicators of a common core construct? Journal of Personality and Social Psychology, 83(3), 693-710. https://doi. org/10.1037//0022-3514.83.3.693

Keller, M. B., Lavori, P. W., Wunder, J., Beardslee, W. R., Schwartz, C. E., \& Roth, J. (1992). Chronic course of anxiety disorders in children and adolescents. Journal of the American Academy of Child and Adolescent Psychiatry, 31(4), 595-599. https://doi.org/ 10.1097/00004583-199207000-00003

Kemp, G. N., Langer, D. A., \& Tompson, M. C. (2016). Childhood mental health: An ecological analysis of the effects of neighborhood characteristics. Journal of Community Psychology, 44(8), 962-979. https://doi.org/10.1002/jcop.21821

Lansford, J. E., Chang, L., Dodge, K. A., Malone, P. S., Oburu, P., Palmérus, K., Bacchini, D., Pastorelli, C., Bombi, A. S., Zelli, A., Tapanya, S., Chaudhary, N., Deater-Deckard, K., Manke, B., \& Quinn, N. (2005). Physical discipline and children's adjustment: Cultural normativeness as a moderator. Child Development, 76(6), 1234-1246. https://doi.org/10.1111/j.1467-8624.2005.00847.x

Lara, R., Vázquez, M. L., Ogallar, A., \& Godoy-Izquierdo, D. (2020). Optimism and social support moderate the indirect relationship between self-efficacy and happiness through mental health in the elderly. Health Psychology Open, 7(2), 2055102920947905. https://doi.org/10.1177/2055102920947905

Lee, H. Y., Kim, I., Nam, S., \& Jeong, J. (2020). Adverse childhood experiences and the associations with depression and anxiety in adolescents. Children and Youth Services Review, 111, 104850. https://doi.org/10.1016/j.childyouth.2020.104850

Li, D. (2012). Multiple ecological risk factors and adolescents' social adaptation: How risks should be modeled and what are their mechanisms [Doctoral dissertation]. South China Normal University. 
Li, H. (2004). The relationship between social support and mental health and personality of college students. Chinese Journal of School Health, 25(3), 263-264.

Lin, C. (2009). Developmental Psychology (2nd ed.). People's Education Press.

Liu, X., Fan, X., \& Shen, J. (2007). Relationship between social support and problem behaviors of the left-home-kids in junior middle school. Psychological Development and Education(03), 98-102.

Liu, X., \& Sun, L. (1997). Analysis of the results of self-rating anxiety scale for 2462 adolescents. Chinese Mental Health Journal, $11(02), 75-77$.

Luo, X., Zheng, R., Jin, X., \& Dai, Y. (2019). Global and chinese perspectives on adolescent health and development. Chin J Sch Health, 40(8), 1126-1130.

MacBeth, A., \& Gumley, A. (2012). Exploring compassion: A metaanalysis of the association between self-compassion and psychopathology. Clinical Psychology Review, 32(6), 545-552. https:// doi.org/10.1016/j.cpr.2012.06.003

McCarthy, C. (2019). Anxiety in teens is rising: What's going on? American Academy of Pediatrics. https://www.healthychildren. org/English/health-issues/conditions/emotional-problems/Pages/ Anxiety-Disorders.aspx

Merrick, M. T., Ford, D. C., Ports, K. A., \& Guinn, A. S. (2018). Prevalence of adverse childhood experiences from the 2011-2014 behavioral risk factor surveillance system in 23 states. JAMA Pediatrics, 172(11), 1038-1044. https://doi.org/10.1001/jamapediat rics.2018.2537

Naismith, I., Zarate Guerrero, S., \& Feigenbaum, J. (2019). Abuse, invalidation, and lack of early warmth show distinct relationships with self-criticism, self-compassion, and fear of self-compassion in personality disorder. Clinical Psychology \& Psychotherapy, 26(3), 350-361. https://doi.org/10.1002/cpp.2357

National Center for Injury Prevention and Control. (2020, March 4). Preventing adverse childhood experiences. https://www.cdc.gov/ violenceprevention/acestudy/fastfact.html?CDC_AA_refVal= https $\% 3 \mathrm{~A} \% 2 \mathrm{~F} \% 2 \mathrm{Fwww} . c d c . g o v \% 2 \mathrm{Fviolenceprevention} \% 2 \mathrm{Fchi}$ ldabuseandneglect $\% 2$ Faces $\% 2$ Ffastfact.html

Neff, K. (2003a). The development and validation of a scale to measure self-compassion. Self and Identity, 2(3), 223-250. https://doi.org/ $10.1080 / 15298860309027$

Neff, K. (2003b). Self-compassion: An alternative conceptualization of a healthy attitude toward oneself. Self and Identity, 2(2), 85-101. https://doi.org/10.1080/15298860309032

Neff, K. (2012). The physiology of self-compassion. https://www.psych ologytoday.com/ca/blog/the-power-self-compassion/201207/thephysiology-self-compassion

Neff, K., \& McGehee, P. (2010). Self-compassion and psychological resilience among adolescents and young adults. Self and Identity, 9(3), 225-240. https://doi.org/10.1080/15298860902979307

Noffsinger, M. A., Pfefferbaum, B., Pfefferbaum, R. L., Sherrib, K., \& Norris, F. H. (2012). The burden of disaster: Part I. Challenges and opportunities within a child's social ecology. International Journal of Emergency Mental Health, 14(1), 3-13.

Oral, R., Ramirez, M., Coohey, C., Nakada, S., Walz, A., Kuntz, A., Benoit, J., \& Peek-Asa, C. (2016). Adverse childhood experiences and trauma informed care: The future of health care. Pediatric Research, 79(1-2), 227-233. https://doi.org/10.1038/pr.2015.197

Pepping, C. A., Davis, P. J., O'Donovan, A., \& Pal, J. (2015). Individual differences in self-compassion: The role of attachment and experiences of parenting in childhood. Self and Identity, 14(1), 104-117. https://doi.org/10.1080/15298868.2014.955050

Polanczyk, G. V., Salum, G. A., Sugaya, L. S., Caye, A., \& Rohde, L. A. (2015). Annual research review: A meta-analysis of the worldwide prevalence of mental disorders in children and adolescents. Journal of Child Psychology and Psychiatry, and
Allied Disciplines, 56(3), 345-365. https://doi.org/10.1111/ jcpp.12381

Raes, F., Pommier, E., Neff, K. D., \& van Gucht, D. (2011). Construction and factorial validation of a short form of the selfcompassion scale. Clinical Psychology \& Psychotherapy, 18(3), 250-255. https://doi.org/10.1002/cpp.702

Rankin, J. L., Lane, D. J., Gibbons, F. X., \& Gerrard, M. (2004). Adolescent self-consciousness: Longitudinal age changes and gender differences in two cohorts. Journal of Research on Adolescence, 14(1), 1-21. https://doi.org/10.1111/j.1532-7795. 2004.01401001.x

Ranøyen, I., Lydersen, S., Larose, T. L., Weidle, B., Skokauskas, N., Thomsen, P. H., Wallander, J., \& Indredavik, M. S. (2018). Developmental course of anxiety and depression from adolescence to young adulthood in a prospective norwegian clinical cohort. European Child \& Adolescent Psychiatry, 27(11), 14131423. https://doi.org/10.1007/s00787-018-1139-7

Reyes Fernández, B., Montenegro Montenegro, E., Knoll, N., \& Schwarzer, R. (2014). Self-efficacy, action control, and social support explain physical activity changes among costa rican older adults. Journal of Physical Activity \& Health, 11(8), 1573-1578. https://doi.org/10.1123/jpah.2013-0175

Rockhill, C., Kodish, I., DiBattisto, C., Macias, M., Varley, C., \& Ryan, S. (2010). Anxiety disorders in children and adolescents. Current Problems in Pediatric and Adolescent Health Care, 40(4), 66-99. https://doi.org/10.1016/j.cppeds.2010.02.002

Rutter, M. (1987). Psychosocial resilience and protective mechanisms. The American Journal of Orthopsychiatry, 57(3), 316331. https://doi.org/10.1111/j.1939-0025.1987.tb03541.x

Saegert, S., \& Evans, G. W. (2003). Poverty, housing niches, and health in the united states. Journal of Social Issues, 59(3), 569-589. https://doi.org/10.1111/1540-4560.00078

Sandler, I. (2001). Quality and ecology of adversity as common mechanisms of risk and resilience. American Journal of Community Psychology, 29(1), 19-61. https://doi.org/10.1023/A: 1005237110505

Sargent, L. D., \& Terry, D. J. (2000). The moderating role of social support in karasek's job strain model. Work \& Stress, 14(3), 245-261. https://doi.org/10.1080/02678370010025568

Spitzer, R. L., Kroenke, K., Williams, J. B., \& Löwe, B. (2006). A brief measure for assessing generalized anxiety disorder: the GAD-7. Archives of internal medicine, 166(10), 1092-1097. https://doi.org/10.1001/archinte.166.10.1092

Steinberg, L. D. (2011). You and your adolescent: The essential guide for ages 10-25 (1st Simon \& Schuster trade pbk. ed.). Simon \& Schuster.

$\mathrm{Su}$, L. (2007). Anxiety disorders in children and adolescents. Journal of New Medicine, 38(04), 214-216\&226.

Tanaka, M., Wekerle, C., Schmuck, M. L., \& Paglia-Boak, A. (2011). The linkages among childhood maltreatment, adolescent mental health, and self-compassion in child welfare adolescents. Child Abuse \& Neglect, 35(10), 887-898. https://doi.org/10.1016/j. chiabu.2011.07.003

Thoits, P. A. (1995). Stress, coping, and social support processes: Where are we? What next? Journal of Health and Social Behavior, Spec No, 53-79.

Vallance, A. K., \& Fernandez, V. (2016). Anxiety disorders in children and adolescents: Aetiology, diagnosis and treatment. Bjpsych Advances, 22(5), 335-344. https://doi.org/10.1192/apt. bp.114.014183

van Dam, N. T., Sheppard, S. C., Forsyth, J. P., \& Earleywine, M. (2011). Self-compassion is a better predictor than mindfulness of symptom severity and quality of life in mixed anxiety and depression. Journal of Anxiety Disorders, 25(1), 123-130. https://doi.org/10.1016/j.janxdis.2010.08.011 
Vanderbilt-Adriance, E., \& Shaw, D. S. (2008). Protective factors and the development of resilience in the context of neighborhood disadvantage. Journal of Abnormal Child Psychology, 36(6), 887-901. https://doi.org/10.1007/s10802-008-9220-1

Wang, G. (2006). Introverting: External representation of psychological mature. Education Science, 22(3), 95-96.

Wang, M., \& Liu, L. (2014). Parental harsh discipline in mainland china: Prevalence, frequency, and coexistence. Child Abuse \& Neglect, 38(6), 1128-1137. https://doi.org/10.1016/j.chiabu.2014. 02.016

Wang, Y., Zhang, W., Peng, J., Mo, B., \& Xiong, S. (2009). The relations of attachment, self-concept and deliberate self-harm in college students. Psychological Exploration, 29(5), 56-61.

Wei, P. (2019, March 5). Hechi ensures that 200,000 poor people are lifted out of poverty this year. Guangxi Daily. http://gxrb.gxrb. com.cn/html/2019-03/05/content_1578712.htm

Winkel, G., Saegert, S., \& Evans, G. W. (2009). An ecological perspective on theory, methods, and analysis in environmental psychology: Advances and challenges. Journal of Environmental Psychology, 29(3), 318-328. Advance online publication. https://doi.org/ 10.1016/J.JENVP.2009.02.005

Wolchik, S. A., Ma, Y., Tein, J.-Y., Sandler, I. N., \& Ayers, T. S. (2008). Parentally bereaved children's grief: Self-system beliefs as mediators of the relations between grief and stressors and caregiverchild relationship quality. Death Studies, 32(7), 597-620. https:// doi.org/10.1080/07481180802215551

Xiao, S. (1994). Theoretical basis and research application of social support scale. Journal of Clinical Psychiatry, 04(02), 98-100.

Yang, H. (2005). Analysis of anxiety and suicide among adolescents. Science of Social Psychology(03), 67-70.

Yang, X., Wang, Y., Li, D., Zhao, L., \& Bao, Z. \& Zhou, Z. (2013). School climate and adolescents' suicidal ideation and suicide attempts: The mediating role of self-esteem. Pyschological Development and Education(05), 541-551.

Yao, R., Zhuang, Y., Han, H., \& Tao, F. (2009). Relationship between life satisfaction and anxiety disorders among adolescents. Chinese Journal of Public Health, 25(5), 541-543.

Ye, D., \& Reyes-Salvail, F. (2014). Adverse childhood experiences among hawai 'I adults: Findings from the 2010 behavioral risk factor survey. Hawai'i Journal of Medicine \& Public Health, 73(6), 181-190.

Zhang, L., Fan, F., Qin, Y., \& Sun, S. (2013). Anxiety and related emotional disorders among the teenagers of rapid urbanization areas. Chinese Journal of Clinical Psychology, 21(3), 434-438.

Zhao, J., Shao, Y., \& Sun, C. (2013). Study on psychological crisis and intervention strategy of junior high school freshmen. Modern Education Science, 1, 77-79.

Zhou, S.-J., Zhang, L.-G., Wang, L.-L., Guo, Z.-C., Wang, J.-Q., Chen, J.-C., Liu, M., Chen, X., \& Chen, J.-X. (2020). Prevalence and socio-demographic correlates of psychological health problems in chinese adolescents during the outbreak of covid-19. European Child \& Adolescent Psychiatry, 29(6), 749-758. https://doi.org/ 10.1007/s00787-020-01541-4

Zimmerman, M. A. (2013). Resiliency theory: A strengths-based approach to research and practice for adolescent health. Health Education \& Behavior: The Official Publication of the Society for Public Health Education, 40(4), 381-383. https://doi.org/10. 1177/1090198113493782

Publisher's note Springer Nature remains neutral with regard to jurisdictional claims in published maps and institutional affiliations. 